\title{
On the Existence of Homoclinic Type Solutions of a Class of Inhomogenous Second Order Hamiltonian Systems
}

\author{
Jakub Ciesielski ${ }^{1} \cdot$ Joanna Janczewska $^{1}$ (D) Nils Waterstraat ${ }^{2}$
}

Received: 19 September 2017 / Revised: 4 June 2019 / Published online: 17 June 2019

(c) The Author(s) 2019

\begin{abstract}
We show the existence of homoclinic type solutions of second order Hamiltonian systems of the type $\ddot{q}(t)+\nabla_{q} V(t, q(t))=f(t)$, where $t \in \mathbb{R}$, the $C^{1}$-smooth potential $V: \mathbb{R} \times \mathbb{R}^{n} \rightarrow$ $\mathbb{R}$ satisfies a relaxed superquadratic growth condition, its gradient is bounded in the time variable, and the forcing term $f: \mathbb{R} \rightarrow \mathbb{R}^{n}$ is sufficiently small in the space of square integrable functions. The idea of our proof is to approximate the original system by timeperiodic ones, with larger and larger time-periods. We prove that the latter systems admit periodic solutions of mountain-pass type, and obtain homoclinic type solutions of the original system from them by passing to the limit (in the topology of almost uniform convergence) when the periods go to infinity.
\end{abstract}

Keywords Homoclinic type solutions · Hamiltonian systems · Approximative method

\section{Introduction}

During the past 2 decades there have been numerous applications of methods from the calculus of variations to find periodic, homoclinic and heteroclinic solutions for Hamiltonian systems. Many of the striking results that have been obtained by variational methods can be found in the well-known monographs of Ambrosetti and Coti Zelati [3], Ekeland [8], Hofer and Zehnder [9], Mawhin and Willem [16], as well as in the review articles of Rabinowitz $[19,20]$.

$\bowtie$ Joanna Janczewska

joanna.janczewska@pg.edu.pl

Jakub Ciesielski

jakub.ciesielski@pg.edu.pl

Nils Waterstraat

waterstraat@web.de

1 Faculty of Applied Physics and Mathematics, Gdansk University of Technology, Narutowicza 11/12, 80-233 Gdańsk, Poland

2 Institut für Mathematik, Naturwissenschaftliche Fakultät II, Martin-Luther-Universität Halle-Wittenberg, Halle (Saale), Germany 
The aim of this paper is to prove the existence of solutions of the second order Hamiltonian system

$$
\left\{\begin{array}{l}
\ddot{q}(t)+\nabla_{q} V(t, q(t))=f(t), \quad t \in \mathbb{R}, \\
\lim _{t \rightarrow \pm \infty} q(t)=\lim _{t \rightarrow \pm \infty} \dot{q}(t)=0,
\end{array}\right.
$$

where the $C^{1}$-smooth potential $V: \mathbb{R} \times \mathbb{R}^{n} \rightarrow \mathbb{R}$ satisfies a relaxed superquadratic growth condition, its gradient $V_{q}: \mathbb{R} \times \mathbb{R}^{n} \rightarrow \mathbb{R}^{n}$ is uniformly bounded in the time variable on every compact subset of $\mathbb{R}^{n}$, and the norm of the forcing term $f: \mathbb{R} \rightarrow \mathbb{R}^{n}$ in the space of square integrable functions is smaller than a bound that we state below in our main theorem.

As homoclinic type solutions are global in time, it is reasonable to use global methods to find them rather than approaches based on their initial value problems. The homogenous systems of (1), i.e. when $f \equiv 0$, have been studied extensively under the assumption of superquadratic or subquadratic growth of the potential $V(t, q)$ as $|q| \rightarrow \infty$. Indeed, there are many results on homoclinic solutions for subquadratic Hamiltonian systems (cf. e.g. [20]). The first variational results for homoclinic solutions of first order Hamiltonian systems with superquadratic growth were found by Coti Zelati et al. [6] for time-periodic Hamiltonians. Corresponding results for second order Hamiltonian systems were obtained in $[7,18]$. Alama and $\mathrm{Li}$ [1] showed that asymptotic periodicity in time actually suffices to get a homoclinic solution, and Serra et al. [21] weakened their periodicity condition to almost periodicity in the sense of Bohr. Finally, Hamiltonian systems with superquadratic non-periodic potentials were investigated for example by Montecchiari and Nolasco [17], Ambrosetti and Badiale [4], and by the second author of this paper in $[11,13,14]$.

Our purpose is to generalize Theorem 1.1 of [5], which deals with the existence of solutions of the inhomogeneous systems (1) under the rather restrictive assumption that the potential $V$ is of the special form

$$
V(t, q)=-\frac{1}{2}|q|^{2}+a(t) G(q),
$$

where $a: \mathbb{R} \rightarrow \mathbb{R}$ is a continuous positive bounded function and $G: \mathbb{R}^{n} \rightarrow \mathbb{R}$ is of class $C^{1}$ and satisfies the Ambrosetti-Rabinowitz superquadratic growth condition. Here, instead, the potential is of the more general form

$$
V(t, q)=-K(t, q)+W(t, q)
$$

with $C^{1}$-smooth potentials $K$ and $W$ such that

$\left(C_{1}\right)$ the maps $\nabla_{q} K$ and $\nabla_{q} W$ are uniformly bounded in the time variable $t \in \mathbb{R}$ on every compact subset of $\mathbb{R}^{n}$,

$\left(C_{2}\right)$ there exist two positive constants $b_{1}, b_{2}$ such that for all $t \in \mathbb{R}$ and $q \in \mathbb{R}^{n}$

$$
b_{1}|q|^{2} \leq K(t, q) \leq b_{2}|q|^{2},
$$

$\left(C_{3}\right) K(t, q) \leq\left(q, \nabla_{q} K(t, q)\right) \leq 2 K(t, q)$ for all $t \in \mathbb{R}$ and $q \in \mathbb{R}^{n}$,

$\left(C_{4}\right) \nabla_{q} W(t, q)=o(|q|)$ as $|q| \rightarrow 0$ uniformly in $t \in \mathbb{R}$,

$\left(C_{5}\right)$ there is a constant $\mu>2$ such that for all $t \in \mathbb{R}$ and $q \in \mathbb{R}^{n} \backslash\{0\}$

$$
0<\mu W(t, q) \leq\left(q, \nabla_{q} W(t, q)\right)
$$

(C6) $m:=\inf \{W(t, q): t \in \mathbb{R} \wedge|q|=1\}>0$.

Here and subsequently, we denote by $(\cdot, \cdot): \mathbb{R}^{n} \times \mathbb{R}^{n} \rightarrow \mathbb{R}$ the standard inner product in $\mathbb{R}^{n}$ and by $|\cdot|$ its induced norm. 
Let us point out that under the above assumptions the Hamiltonian system (1) has the trivial solution when the forcing term $f$ vanishes. Therefore it is reasonable to suppose that homoclinic type solutions exist when $f$ is sufficiently small. Our main result affirms this hypothesis and it also gives an answer to the question how large the forcing term can be.

Theorem 1.1 Set $M:=\sup \{W(t, q): t \in \mathbb{R} \wedge|q|=1\}$ and $\bar{b}_{1}:=\min \left\{1,2 b_{1}\right\}$. Let us assume that $M<\frac{1}{2} \bar{b}_{1}$ and $\left(C_{1}\right)-\left(C_{6}\right)$ are satisfied. If the forcing term $f$ is continuous, bounded, and moreover

$$
\left(\int_{-\infty}^{\infty}|f(t)|^{2} d t\right)^{\frac{1}{2}}<\frac{\sqrt{2}}{4}\left(\bar{b}_{1}-2 M\right),
$$

then the inhomogenous system (1) possesses at least one solution.

The idea of our proof, which we give in the following second section, is to approximate the original system (1) by time-periodic ones, with larger and larger time-periods. We show that the approximating systems admit periodic solutions of mountain-pass type, and obtain a homoclinic type solution of the original system from them by passing to the limit (in the topology of almost uniform convergence) when the periods go to infinity. Finally, we discuss some examples of Theorem 1.1 in Sect. 3.

\section{Proof of Theorem 1.1}

For each $k \in \mathbb{N}$, let $E_{k}=W_{2 k}^{1,2}\left(\mathbb{R}, \mathbb{R}^{n}\right)$ be the Sobolev space of $2 k$-periodic functions on $\mathbb{R}$ with values in $\mathbb{R}^{n}$ and the standard norm

$$
\|q\|_{E_{k}}=\left(\int_{-k}^{k}\left(|\dot{q}(t)|^{2}+|q(t)|^{2}\right) d t\right)^{\frac{1}{2}} .
$$

We begin with the following estimate that is crucial in the main part of our proof below.

Lemma 2.1 For every $\zeta \in \mathbb{R}$ and $q \in E_{k}$ we have

$$
\int_{-k}^{k} W(t, \zeta q(t)) d t \geq m|\zeta|^{\mu} \int_{-k}^{k}|q(t)|^{\mu} d t-2 k m .
$$

Proof Note at first that the assertion is obviously true if $q=0$ or $\zeta=0$. Hence we can assume in the rest of the proof that $\zeta \neq 0$ and $q \neq 0$. Then it follows from $\left(C_{5}\right)$ that, for every $q \neq 0$ and $t \in \mathbb{R}$, the function $z:(0,+\infty) \rightarrow \mathbb{R}$ defined by

$$
z(\zeta)=W\left(t, \frac{q}{\zeta}\right) \zeta^{\mu}
$$

is non-increasing. Hence, for every $t \in \mathbb{R}$,

$$
W(t, q) \leq W\left(t, \frac{q}{|q|}\right)|q|^{\mu}, \quad \text { if } 0<|q| \leq 1
$$

and

$$
W(t, q) \geq W\left(t, \frac{q}{|q|}\right)|q|^{\mu}, \quad \text { if }|q| \geq 1 .
$$


We now fix $\zeta \in \mathbb{R} \backslash\{0\}, q \in E_{k} \backslash\{0\}$ and set

$$
\begin{aligned}
& A_{k}=\{t \in[-k, k]:|\zeta q(t)| \leq 1\}, \\
& B_{k}=\{t \in[-k, k]:|\zeta q(t)| \geq 1\} .
\end{aligned}
$$

By (4), we get

$$
\begin{aligned}
\int_{-k}^{k} W(t, \zeta q(t)) d t & \geq \int_{B_{k}} W(t, \zeta q(t)) d t \geq \int_{B_{k}} W\left(t, \frac{\zeta q(t)}{|\zeta q(t)|}\right)|\zeta q(t)|^{\mu} d t \\
& \geq m \int_{B_{k}}|\zeta q(t)|^{\mu} d t \geq m \int_{-k}^{k}|\zeta q(t)|^{\mu} d t-m \int_{A_{k}}|\zeta q(t)|^{\mu} d t \\
& \geq m|\zeta|^{\mu} \int_{-k}^{k}|q(t)|^{\mu} d t-2 k m
\end{aligned}
$$

which completes the proof.

Further, to prove Theorem 1.1, we need the following approximative method.

Theorem 2.2 (Approximative Method, [15]) Let $f: \mathbb{R} \rightarrow \mathbb{R}^{n}$ be a non-trivial, bounded, continuous and square integrable map. Assume that $V: \mathbb{R} \times \mathbb{R}^{n} \rightarrow \mathbb{R}$ is a $C^{1}$-smooth potential such that $\nabla_{q} V: \mathbb{R} \times \mathbb{R}^{n} \rightarrow \mathbb{R}^{n}$ is uniformly bounded in t on every compact subset of $\mathbb{R}^{n}$, i.e.

$$
\forall L>0 \exists C>0 \quad \forall q \in \mathbb{R}^{n} \quad \forall t \in \mathbb{R} \quad|q| \leq L \Rightarrow\left|\nabla_{q} V(t, q)\right| \leq C .
$$

Suppose that for each $k \in \mathbb{N}$ the boundary value problem

$$
\left\{\begin{array}{l}
\ddot{q}(t)+\nabla_{q} V_{k}(t, q(t))=f_{k}(t), \\
q(-k)-q(k)=\dot{q}(-k)-\dot{q}(k)=0,
\end{array}\right.
$$

where $f_{k}: \mathbb{R} \rightarrow \mathbb{R}^{n}$ standsfor the $2 k$-periodic extension of $\left.f\right|_{[-k, k)}$ to $\mathbb{R}$ and $V_{k}: \mathbb{R} \times \mathbb{R}^{n} \rightarrow \mathbb{R}$ denotes the $2 k$-periodic extension of $\left.V\right|_{[-k, k) \times \mathbb{R}^{n}}$ to $\mathbb{R} \times \mathbb{R}^{n}$, has a periodic solution $q_{k} \in E_{k}$ and $\left\{\left\|q_{k}\right\|_{E_{k}}\right\}_{k \in \mathbb{N}}$ is a bounded sequence in $\mathbb{R}$. Then there exists a subsequence $\left\{q_{k_{j}}\right\}_{j \in \mathbb{N}}$ converging in the topology of $C_{\text {loc }}^{2}\left(\mathbb{R}, \mathbb{R}^{n}\right)$ to a function $q \in W^{1,2}\left(\mathbb{R}, \mathbb{R}^{n}\right)$ which is a solution of

$$
\ddot{q}(t)+\nabla_{q} V(t, q(t))=f(t), \quad t \in \mathbb{R} .
$$

The approximative method was introduced by Rabinowitz [18] for homogenous second order Hamiltonian systems with a time-periodic potential. Later, the second author of this paper extended it to inhomogenous time-periodic Hamiltonian systems (see $[10,12]$ ), and more recently, Robert Krawczyk generalized it to the case of aperiodic potentials.

Let us now consider for $k \in \mathbb{N}$ the boundary value problems

$$
\left\{\begin{array}{l}
\ddot{q}(t)-\nabla_{q} K_{k}(t, q(t))+\nabla_{q} W_{k}(t, q(t))=f_{k}(t), \\
q(-k)-q(k)=\dot{q}(-k)-\dot{q}(k)=0,
\end{array}\right.
$$

where $f_{k}: \mathbb{R} \rightarrow \mathbb{R}^{n}$ stands for the $2 k$-periodic extension of $\left.f\right|_{[-k, k)}$ to $\mathbb{R}$, and $K_{k}: \mathbb{R} \times \mathbb{R}^{n} \rightarrow$ $\mathbb{R}, W_{k}: \mathbb{R} \times \mathbb{R}^{n} \rightarrow \mathbb{R}$ are the $2 k$-periodic extensions of $\left.K\right|_{[-k, k) \times \mathbb{R}^{n}}$ and $\left.W\right|_{[-k, k) \times \mathbb{R}^{n}}$ to $\mathbb{R} \times \mathbb{R}^{n}$.

As we have already mentioned in the introduction, our proof consists of two steps. First, we show the existence of solutions of (5), and second, we use Theorem 2.2 to find a solution of (1). 
For our first step, let us consider the functionals $I_{k}: E_{k} \rightarrow \mathbb{R}$ given by

$$
I_{k}(q)=\int_{-k}^{k}\left(\frac{1}{2}|\dot{q}(t)|^{2}+K_{k}(t, q(t))-W_{k}(t, q(t))\right) d t+\int_{-k}^{k}\left(f_{k}(t), q(t)\right) d t .
$$

Standard arguments show that $I_{k} \in C^{1}\left(E_{k}, \mathbb{R}\right)$, and

$$
I_{k}^{\prime}(q) v=\int_{-k}^{k}\left((\dot{q}(t), \dot{v}(t))+\left(\nabla_{q} K_{k}(t, q(t))-\nabla_{q} W_{k}(t, q(t)), v(t)\right)\right) d t+\int_{-k}^{k}\left(f_{k}(t), v(t)\right) d t .
$$

Moreover, the critical points of the functional $I_{k}$ are classical $2 k$-periodic solutions of (5), and we now show their existence by using the Mountain Pass Theorem. Let us recall the latter result before proceeding with our proof.

Theorem 2.3 (Mountain Pass Theorem, [2]) Let $E$ be a real Banach space and $I: E \rightarrow \mathbb{R}$ a $C^{1}$-smooth functional. If I satisfies the following conditions:

(i) $I(0)=0$,

(ii) every sequence $\left\{u_{j}\right\}_{j \in \mathbb{N}}$ in E such that $\left\{I\left(u_{j}\right)\right\}_{j \in \mathbb{N}}$ is bounded in $\mathbb{R}$ and $I^{\prime}\left(u_{j}\right) \rightarrow 0$ in $E^{*}$ as $j \rightarrow+\infty$ contains a convergent subsequence (the Palais-Smale condition),

(iii) there exist constants $\rho, \alpha>0$ such that $\left.I\right|_{\partial B_{\rho}(0)} \geq \alpha$,

(iv) there exists $e \in E \backslash \bar{B}_{\rho}(0)$ such that $I(e) \leq 0$,

where $B_{\rho}(0)$ is the open ball of radius $\rho$ about 0 in $E$, then I possesses a critical value $c \geq \alpha$ given by

$$
c=\inf _{g \in \Gamma} \max _{s \in[0,1]} I(g(s)),
$$

where

$$
\Gamma=\{g \in C([0,1], E): g(0)=0, g(1)=e\} .
$$

We now denote by $L_{2 k}^{\infty}\left(\mathbb{R}, \mathbb{R}^{n}\right)$ the space of $2 k$-periodic essentially bounded functions from $\mathbb{R}$ into $\mathbb{R}^{n}$ equipped with the norm

$$
\|q\|_{L_{2 k}^{\infty}}=\operatorname{ess} \sup \{|q(t)|: t \in[-k, k]\} .
$$

It is well known that for each $k \in \mathbb{N}$ and $q \in E_{k}$

$$
\|q\|_{L_{2 k}^{\infty}} \leq \sqrt{2}\|q\|_{E_{k}} .
$$

The proof of (9) can be found for example in [10] (see Fact 2.8, p. 385).

Furthermore, we will write $L_{2 k}^{2}\left(\mathbb{R}, \mathbb{R}^{n}\right)$ for the Hilbert space of $2 k$-periodic functions on $\mathbb{R}$ with values in $\mathbb{R}^{n}$ and with the norm

$$
\|q\|_{L_{2 k}^{2}}=\left(\int_{-k}^{k}|q(t)|^{2} d t\right)^{\frac{1}{2}} .
$$

Note that by (2),

$$
\left\|f_{k}\right\|_{L_{2 k}^{2}}<\frac{\sqrt{2}}{4}\left(\bar{b}_{1}-2 M\right)
$$

The following lemma shows the existence of a solution of (5) and is the main part of the first step of our proof. 
Lemma 2.4 For each $k \in \mathbb{N}$, the functional $I_{k}$ has a critical value of mountain pass type.

Proof We let $k \in \mathbb{N}$ be fixed and note at first that it is evident by $\left(C_{2}\right)$ and $\left(C_{5}\right)$ that $I_{k}(0)=0$, which shows (i) in Theorem 2.3.

For checking the Palais-Smale condition (ii), we consider a sequence $\left\{u_{j}\right\}_{j \in \mathbb{N}} \subset E_{k}$ such that $\left\{I_{k}\left(u_{j}\right)\right\}_{j \in \mathbb{N}}$ is bounded in $\mathbb{R}$ and $I_{k}^{\prime}\left(u_{j}\right) \rightarrow 0$ in $E_{k}^{*}$ as $j \rightarrow \infty$. Then there exists a constant $C_{k}>0$ such that for all $j \in \mathbb{N}$

$$
\left|I_{k}\left(u_{j}\right)\right| \leq C_{k}
$$

and

$$
\left\|I_{k}^{\prime}\left(u_{j}\right)\right\|_{E_{k}^{*}} \leq C_{k} .
$$

Now, we will first show that $\left\{u_{j}\right\}_{j \in \mathbb{N}}$ is bounded in the Hilbert space $E_{k}$. Using (6) and $\left(C_{5}\right)$ we get

$$
\begin{aligned}
2 I_{k}\left(u_{j}\right) \geq & \int_{-k}^{k}\left(\left|\dot{u}_{j}(t)\right|^{2}+2 K_{k}\left(t, u_{j}(t)\right)\right) d t-\frac{2}{\mu} \int_{-k}^{k}\left(\nabla_{q} W_{k}\left(t, u_{j}(t)\right), u_{j}(t)\right) d t \\
& +2 \int_{-k}^{k}\left(f_{k}(t), u_{j}(t)\right) d t .
\end{aligned}
$$

From (7) and $\left(C_{3}\right)$ it follows that

$$
\begin{aligned}
I_{k}^{\prime}\left(u_{j}\right) u_{j} \leq & \int_{-k}^{k}\left(\left|\dot{u}_{j}(t)\right|^{2}+2 K_{k}\left(t, u_{j}(t)\right)\right) d t-\int_{-k}^{k}\left(\nabla_{q} W_{k}\left(t, u_{j}(t)\right), u_{j}(t)\right) d t \\
& +\int_{-k}^{k}\left(f_{k}(t), u_{j}(t)\right) d t .
\end{aligned}
$$

Thus

$$
\begin{aligned}
2 I_{k}\left(u_{j}\right)-\frac{2}{\mu} I_{k}^{\prime}\left(u_{j}\right) u_{j} \geq & \left(1-\frac{2}{\mu}\right) \int_{-k}^{k}\left(\left|\dot{u}_{j}(t)\right|^{2}+2 K_{k}\left(t, u_{j}(t)\right)\right) d t \\
& +\left(2-\frac{2}{\mu}\right) \int_{-k}^{k}\left(f_{k}(t), u_{j}(t)\right) d t
\end{aligned}
$$

and by $\left(C_{2}\right)$ we have

$$
2 I_{k}\left(u_{j}\right)-\frac{2}{\mu} I_{k}^{\prime}\left(u_{j}\right) u_{j} \geq\left(1-\frac{2}{\mu}\right) \bar{b}_{1}\left\|u_{j}\right\|_{E_{k}}^{2}+\left(2-\frac{2}{\mu}\right) \int_{-k}^{k}\left(f_{k}(t), u_{j}(t)\right) d t .
$$

Finally, aplying the Hölder inequality, as well as (10), (11) and (12), we obtain

$$
\left(1-\frac{2}{\mu}\right) \bar{b}_{1}\left\|u_{j}\right\|_{E_{k}}^{2}-\frac{2 C_{k}}{\mu}\left\|u_{j}\right\|_{E_{k}}-\frac{\sqrt{2}}{4}\left(\bar{b}_{1}-2 M\right)\left(2-\frac{2}{\mu}\right)\left\|u_{j}\right\|_{E_{k}}-2 C_{k} \leq 0 .
$$

Since $\mu>2$ we conclude that $\left\{u_{j}\right\}$ is bounded.

Going to a subsequence if necessary, we can assume that there exists a function $u \in E_{k}$ such that $u_{j} \rightarrow u$ weakly in $E_{k}$ as $j \rightarrow+\infty$. Hence $u_{j} \rightarrow u$ uniformly on $[-k, k]$, which implies that

$$
\begin{aligned}
& \left(I_{k}^{\prime}\left(u_{j}\right)-I_{k}^{\prime}(u)\right)\left(u_{j}-u\right) \rightarrow 0, \\
& \left\|u_{j}-u\right\|_{L_{2 k}^{2}} \rightarrow 0
\end{aligned}
$$


and

$$
\begin{aligned}
& \int_{-k}^{k}\left(\nabla_{q} K_{k}\left(t, u_{j}(t)\right)-\nabla_{q} W_{k}\left(t, u_{j}(t)\right), u_{j}(t)-u(t)\right) d t \\
& -\int_{-k}^{k}\left(\nabla_{q} K_{k}(t, u(t))-\nabla_{q} W_{k}(t, u(t)), u_{j}(t)-u(t)\right) d t \rightarrow 0
\end{aligned}
$$

as $j \rightarrow+\infty$. On the other hand, it is readily seen that

$$
\begin{aligned}
\left\|\dot{u}_{j}-\dot{u}\right\|_{L_{2 k}^{2}}^{2}= & \left(I_{k}^{\prime}\left(u_{j}\right)-I_{k}^{\prime}(u)\right)\left(u_{j}-u\right) \\
& -\int_{-k}^{k}\left(\nabla_{q} K_{k}\left(t, u_{j}(t)\right)-\nabla_{q} W_{k}\left(t, u_{j}(t)\right), u_{j}(t)-u(t)\right) d t \\
& +\int_{-k}^{k}\left(\nabla_{q} K_{k}(t, u(t))-\nabla_{q} W_{k}(t, u(t)), u_{j}(t)-u(t)\right) d t,
\end{aligned}
$$

and consequently

$$
\left\|\dot{u}_{j}-\dot{u}\right\|_{L_{2 k}^{2}} \rightarrow 0 .
$$

By (13) and (14), we see that $\left\|u_{j}-u\right\|_{E_{k}} \rightarrow 0$, and thus $I_{k}$ satisfies the Palais-Smale condition.

To show (iii), we set

$$
\rho=\frac{\sqrt{2}}{2} .
$$

Assume that $q \in \partial B_{\rho}(0) \subset E_{k}$. Then $\|q\|_{L_{2 k}^{\infty}}>0$ and $\|q\|_{L_{2 k}^{\infty}} \leq 1$ by (9). Let

$$
D_{k}=\{t \in[-k, k]: 0<|q(t)| \leq 1\} .
$$

Thus, we can apply (3) to obtain

$$
\begin{aligned}
\int_{-k}^{k} W(t, q(t)) d t & =\int_{D_{k}} W(t, q(t)) d t \leq \int_{D_{k}} W\left(t, \frac{q(t)}{|q(t)|}\right)|q(t)|^{\mu} d t \\
& \leq M \int_{D_{k}}|q(t)|^{2} d t=M \int_{-k}^{k}|q(t)|^{2} d t \leq M\|q\|_{E_{k}}^{2}=\frac{1}{2} M .
\end{aligned}
$$

From this, $\left(C_{2}\right)$ and (2), we get

$$
\begin{aligned}
I_{k}(q) & \geq \frac{1}{2} \bar{b}_{1}\|q\|_{E_{k}}^{2}-\frac{1}{2} M-\left\|f_{k}\right\|_{L_{2 k}^{2}}\|q\|_{E_{k}} \\
& \geq \frac{1}{4}\left(\bar{b}_{1}-2 M\right)-\frac{\sqrt{2}}{2}\|f\|_{L^{2}} \\
& =\frac{\sqrt{2}}{2}\left(\frac{\sqrt{2}}{4}\left(\bar{b}_{1}-2 M\right)-\|f\|_{L^{2}}\right) \equiv \alpha>0 .
\end{aligned}
$$

To complete the proof, we have to show (iv), i.e. we need to find $e_{k} \in E_{k}$ such that $\left\|e_{k}\right\|_{E_{k}}>\rho$ and $I_{k}\left(e_{k}\right) \leq 0$.

Let

$$
\bar{b}_{2}=\max \left\{1,2 b_{2}\right\}
$$


Combining (6) and Lemma 2.1 gives

$$
I_{k}(\zeta q) \leq \frac{\bar{b}_{2} \zeta^{2}}{2}\|q\|_{E_{k}}^{2}-m|\zeta|^{\mu} \int_{-k}^{k}|q(t)|^{\mu} d t+|\zeta| \cdot\left\|f_{k}\right\|_{L_{2 k}^{2}}\|q\|_{E_{k}}+2 k m
$$

for all $\zeta \in \mathbb{R} \backslash\{0\}$ and $q \in E_{k} \backslash\{0\}$.

We now let $Q \in E_{1}$ be such that $Q \neq 0$ and $Q(-1)=Q(1)=0$. It follows from (16) that $\|\zeta Q\|_{E_{1}}>\rho$ and $I_{1}(\zeta Q)<0$ for $\zeta \in \mathbb{R} \backslash\{0\}$ large enough. Hence, if we define $e_{1}(t)=\zeta Q(t)$ and for each $k \geq 2$,

$$
e_{k}(t)= \begin{cases}e_{1}(t) & \text { for } t \in[-1,1], \\ 0 & \text { for } t \in[-k,-1) \cup(1, k],\end{cases}
$$

then $e_{k} \in E_{k}$, and $\left\|e_{k}\right\|_{E_{k}}=\left\|e_{1}\right\|_{E_{1}}>\rho$ as well as $I_{k}\left(e_{k}\right)=I_{1}\left(e_{1}\right)<0$.

In summary, it follows from Theorem 2.3 that the action functional $I_{k}$ has a critical value $c_{k} \geq \alpha$ given by

$$
c_{k}=\inf _{g \in \Gamma_{k}} \max _{s \in[0,1]} I_{k}(g(s))
$$

where

$$
\Gamma_{k}=\left\{g \in C\left([0,1], E_{k}\right): g(0)=0, g(1)=e_{k}\right\} .
$$

In what follows, we let $q_{k}$ be a critical point for the corresponding critical value $c_{k}$ that we have found in Lemma 2.4. The functions $q_{k}, k \in \mathbb{N}$, are solutions of (5) and as second step of our proof of Theorem 1.1, we now want to apply Theorem 2.2 to this sequence of functions.

Lemma 2.5 The sequence $\left\{\left\|q_{k}\right\|_{E_{k}}\right\}_{k \in \mathbb{N}} \subset \mathbb{R}$ is bounded.

Proof We set

$$
M_{0}=\max _{s \in[0,1]} I_{1}\left(s e_{1}\right) .
$$

and conclude from (17) and (18) that

$$
c_{k} \leq M_{0}
$$

for each $k \in \mathbb{N}$. By assumption,

$$
\begin{aligned}
c_{k}= & I_{k}\left(q_{k}\right)=I_{k}\left(q_{k}\right)-\frac{1}{2} I_{k}^{\prime}\left(q_{k}\right) q_{k}=\int_{-k}^{k}\left(K_{k}\left(t, q_{k}(t)\right)-\frac{1}{2}\left(\nabla_{q} K_{k}\left(t, q_{k}(t)\right), q_{k}(t)\right)\right) d t \\
& +\int_{-k}^{k}\left(\frac{1}{2}\left(\nabla_{q} W_{k}\left(t, q_{k}(t)\right), q_{k}(t)\right)-W_{k}\left(t, q_{k}(t)\right)\right) d t+\frac{1}{2} \int_{-k}^{k}\left(f_{k}(t), q_{k}(t)\right) d t .
\end{aligned}
$$

Applying $\left(C_{3}\right)$ and $\left(C_{5}\right)$ we obtain

$$
c_{k} \geq\left(\frac{\mu}{2}-1\right) \int_{-k}^{k} W_{k}\left(t, q_{k}(t)\right) d t+\frac{1}{2} \int_{-k}^{k}\left(f_{k}(t), q_{k}(t)\right) d t .
$$

Furthermore, it follows from (6) and $\left(C_{2}\right)$ that

$$
\int_{-k}^{k} W_{k}\left(t, q_{k}(t)\right) d t \geq \frac{1}{2} \bar{b}_{1}\left\|q_{k}\right\|_{E_{k}}^{2}+\int_{-k}^{k}\left(f_{k}(t), q_{k}(t)\right) d t-I_{k}\left(q_{k}\right) .
$$


Using that $I_{k}\left(q_{k}\right)=c_{k}$, the previous two inequalities give

$$
\frac{1}{2} \bar{b}_{1}\left\|q_{k}\right\|_{E_{k}}^{2}-\frac{\mu-1}{\mu-2}\left\|f_{k}\right\|_{L_{2 k}^{2}}\left\|q_{k}\right\|_{E_{k}}-\frac{\mu}{\mu-2} c_{k} \leq 0,
$$

which implies by (2) and (19) that

$$
\frac{1}{2} \bar{b}_{1}\left\|q_{k}\right\|_{E_{k}}^{2}-\frac{\sqrt{2}}{4}\left(\bar{b}_{1}-2 M\right) \frac{\mu-1}{\mu-2}\left\|q_{k}\right\|_{E_{k}}-\frac{\mu}{\mu-2} M_{0} \leq 0 .
$$

Hence there is $M_{1}>0$ such that for each $k \in \mathbb{N}$,

$$
\left\|q_{k}\right\|_{E_{k}} \leq M_{1} .
$$

Now, using Theorem 2.2 we see that there exists a solution $q: \mathbb{R} \rightarrow \mathbb{R}^{n}$ of (1) such that $q(t) \rightarrow 0$ as $|t| \rightarrow \infty$.

All what is left to show for the proof of Theorem 1.1 is that actually $\dot{q}(t) \rightarrow 0$ as $|t| \rightarrow \infty$. This, however, follows from the inequality

$$
|\dot{q}(t)| \leq \sqrt{2}\left(\int_{t-\frac{1}{2}}^{t+\frac{1}{2}}\left(|\dot{q}(s)|^{2}+|\ddot{q}(s)|^{2}\right) d s\right)^{\frac{1}{2}}, \quad t \in \mathbb{R},
$$

which can be found in [10] [Inequality (28), p. 385]. Indeed, we just need to note that by (1), $\left(C_{2}\right),\left(C_{4}\right)$ and $(2)$

$$
\int_{t-\frac{1}{2}}^{t+\frac{1}{2}}|\ddot{q}(s)|^{2} d s \rightarrow 0, \quad|t| \rightarrow \infty .
$$

If now $|t|$ goes to $\infty$ in (20) we see that $|\dot{q}(t)| \rightarrow 0$ as $|t| \rightarrow \infty$. Consequently, $q$ is a solution of (1) and the proof of Theorem 1.1 is complete.

\section{One-Dimensional Examples}

In this section we present examples for $n=1$ satisfying the assumptions of Theorem 1.1, and the graphs of their approximating solutions $q_{k}$ of (5) for increasing values of $k$.

Example 3.1 Consider $K: \mathbb{R} \times \mathbb{R} \rightarrow \mathbb{R}, W: \mathbb{R} \times \mathbb{R} \rightarrow \mathbb{R}$ and $f: \mathbb{R} \rightarrow \mathbb{R}$ given by

$$
\begin{aligned}
K(t, q) & =\frac{t^{2}+1}{t^{2}+2} q^{2}, \\
W(t, q) & =\frac{t^{2}+12}{3 t^{2}+27} q^{4}
\end{aligned}
$$

and

$$
f(t)=\frac{1}{36} e^{-t^{2}}
$$

where $t, q \in \mathbb{R}$. One can easily check that $K, W$ and $f$ satisfy the assumptions of Theorem 1.1. The Figs. 1, 2 and 3 show the graphs of numerical solutions $q_{k}$ of (5) for $k=57,100,250$. 


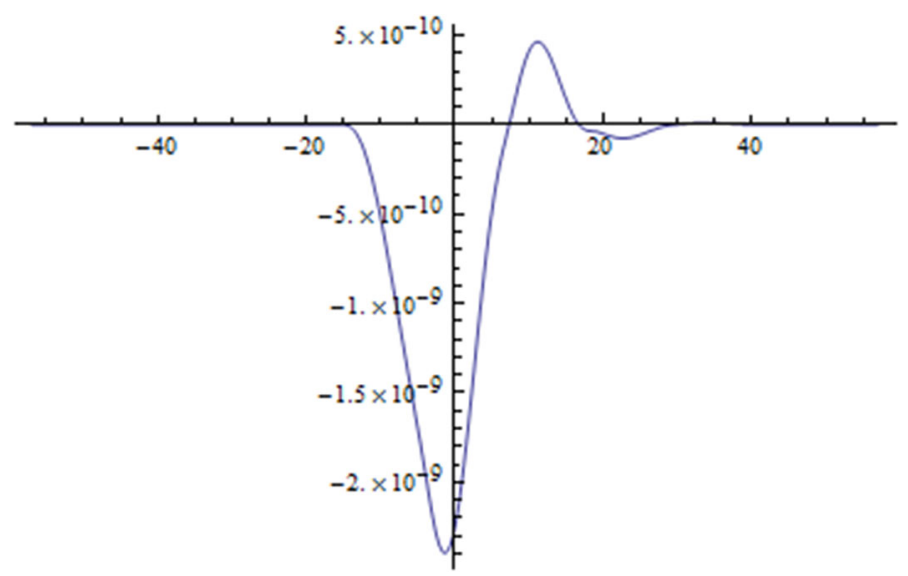

Fig. 1 A numerical solution of (5) for $k=57$ in Example 1

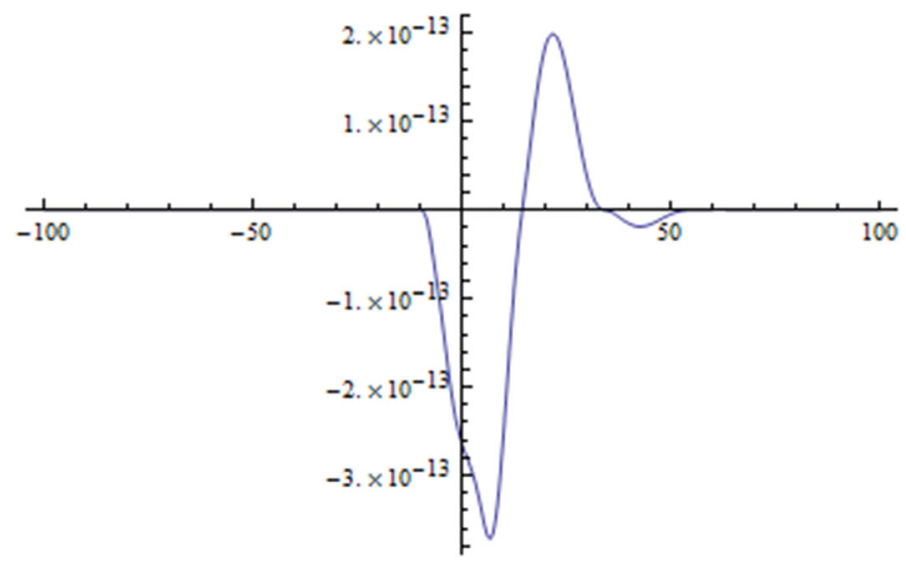

Fig. 2 A numerical solution of (5) for $k=100$ in Example 1

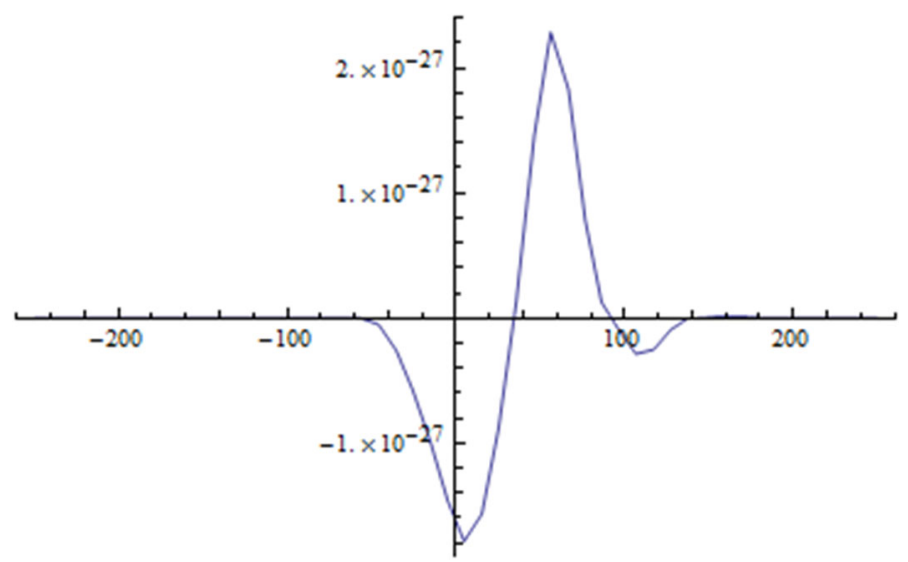

Fig. 3 A numerical solution of (5) for $k=250$ in Example 1 


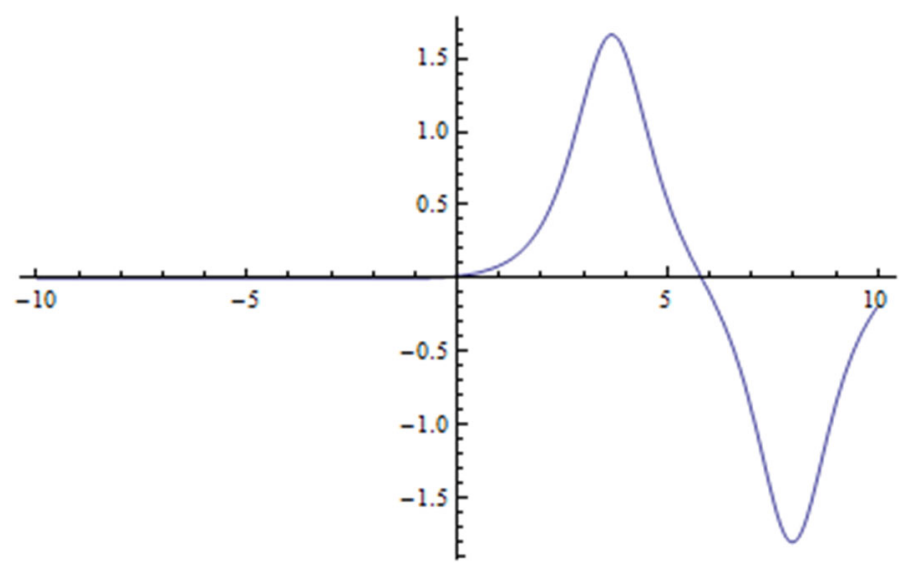

Fig. 4 A numerical solution of (5) for $k=10$ in Example 2

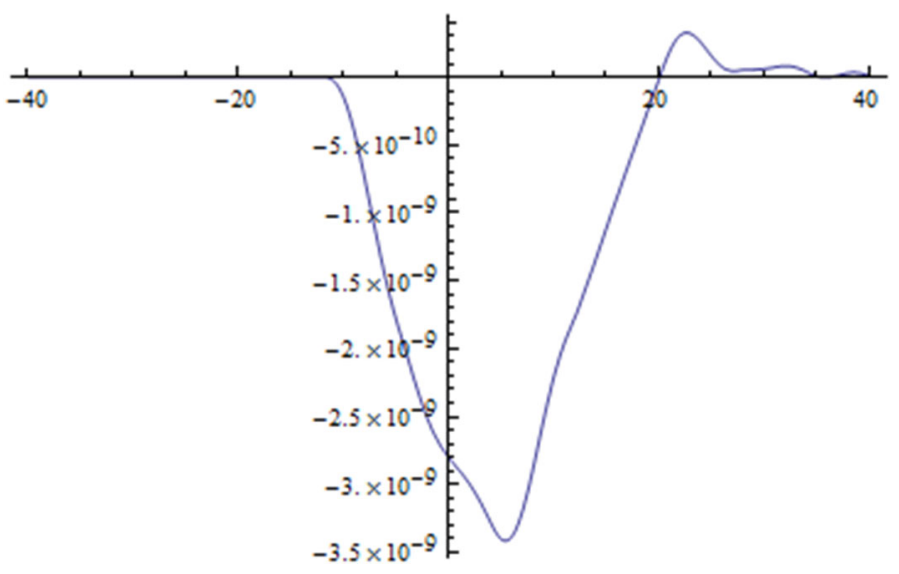

Fig. 5 A numerical solution of (5) for $k=40$ in Example 2

Example 3.2 Let $K: \mathbb{R} \times \mathbb{R} \rightarrow \mathbb{R}, W: \mathbb{R} \times \mathbb{R} \rightarrow \mathbb{R}$ and $f: \mathbb{R} \rightarrow \mathbb{R}$ be given by

$$
\begin{aligned}
K(t, q) & =\left(\frac{1}{8} \sin (t)+\frac{1}{8} \sin (\sqrt{2} t)+\frac{3}{4}\right) q^{2}, \\
W(t, q) & =\frac{1}{4} q^{4}
\end{aligned}
$$

and

$$
f(t)=\frac{1}{32} e^{-t^{2}}
$$

where $t, q \in \mathbb{R}$. It is immediate that $K, W$ and $f$ satisfy the assumptions of Theorem 1.1. The Figs. 4, 5 and 6 show the graphs of numerical solutions $q_{k}$ of (5) for $k=10,40,160$.

Example 3.3 Consider $K: \mathbb{R} \times \mathbb{R} \rightarrow \mathbb{R}, W: \mathbb{R} \times \mathbb{R} \rightarrow \mathbb{R}$ and $f: \mathbb{R} \rightarrow \mathbb{R}$ given by

$$
K(t, q)=q^{2},
$$




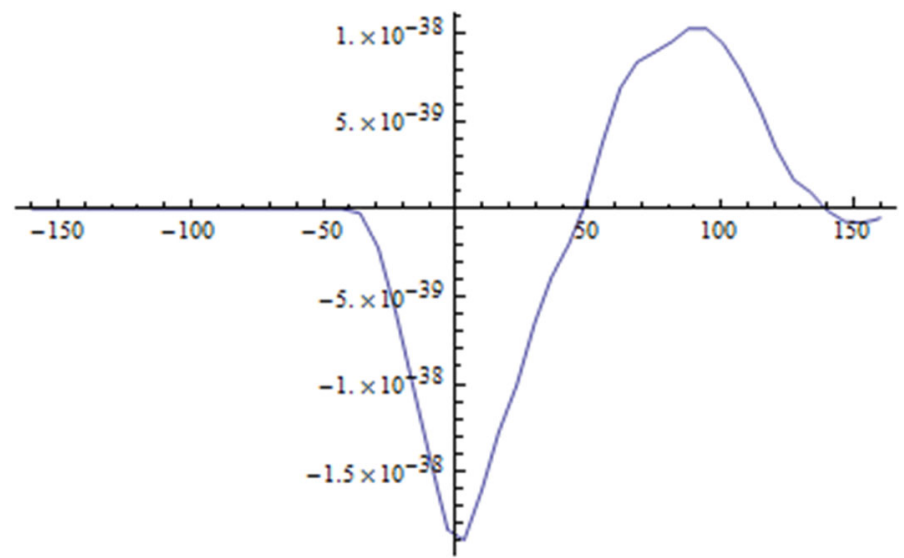

Fig. 6 A numerical solution of (5) for $k=160$ in Example 2

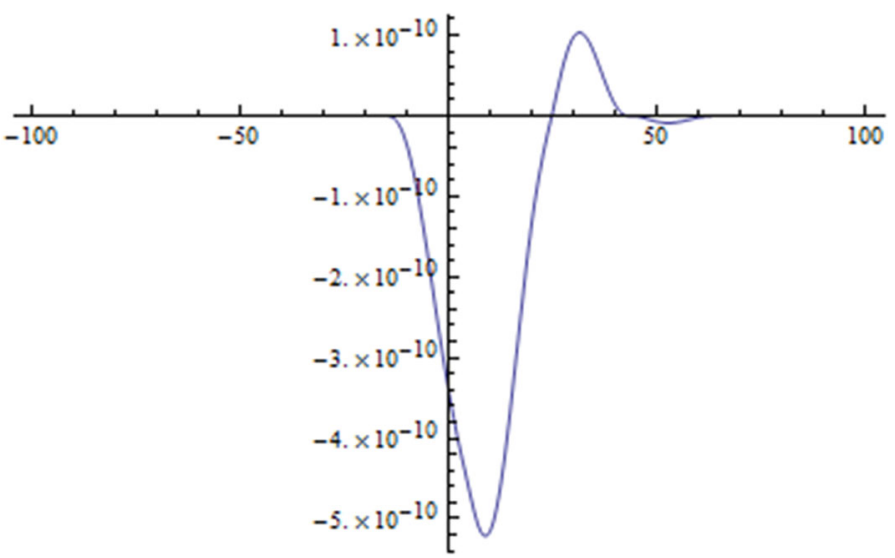

Fig. 7 A numerical solution of (5) for $k=100$ in Example 3

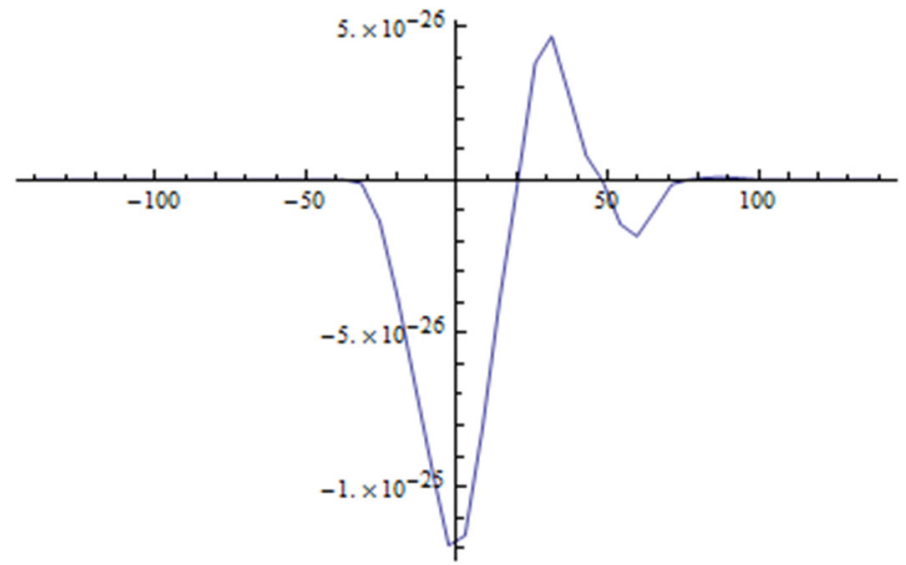

Fig. 8 A numerical solution of (5) for $k=140$ in Example 3 


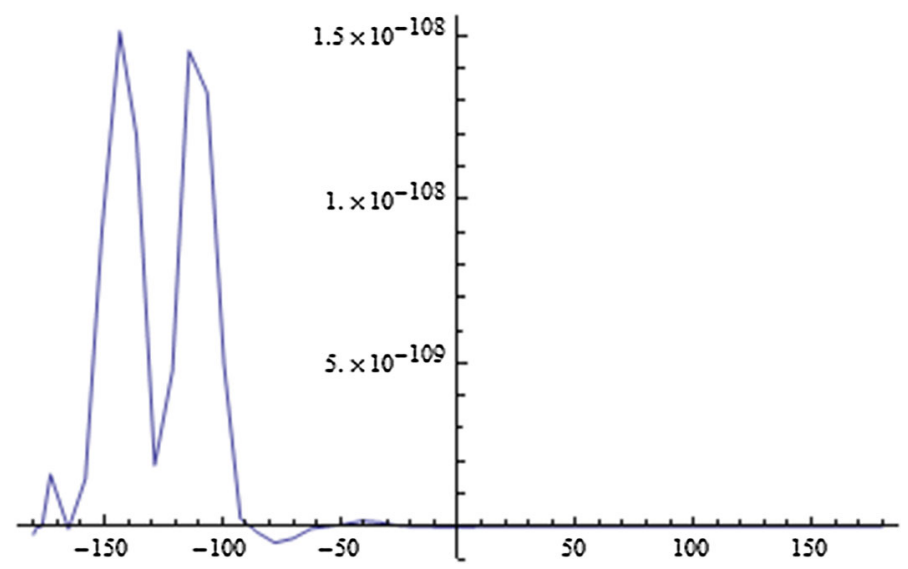

Fig. 9 A numerical solution of (5) for $k=180$ in Example 3

$$
W(t, q)=\frac{10}{33} q^{4}\left(\operatorname{arctg}^{2}\left(\frac{q^{2}}{t^{2}+1}\right)+1\right)
$$

and

$$
f(t)=\frac{1+t^{2}}{10} e^{-t^{2}},
$$

where $t, q \in \mathbb{R}$. Again, it is readily seen that $K, W$ and $f$ satisfy the assumptions of Theorem 1.1. The Figs. 7, 8 and 9 show the graphs of numerical solutions $q_{k}$ of (5) for $k=100,140,180$.

Acknowledgements The second and the third authors were partially supported by Grant PPP-PL No. 57217076 of DAAD and MNiSW, and partially by Grant BEETHOVEN 2 of the National Science Centre, Poland, No. 2016/23/G/ST1/04081.

Open Access This article is distributed under the terms of the Creative Commons Attribution 4.0 International License (http://creativecommons.org/licenses/by/4.0/), which permits unrestricted use, distribution, and reproduction in any medium, provided you give appropriate credit to the original author(s) and the source, provide a link to the Creative Commons license, and indicate if changes were made.

\section{References}

1. Alama, S., Li, Y.Y.: Existence of solutions for semilinear elliptic equations with indefinite linear part. J. Differ. Equ. 96, 88-115 (1992)

2. Ambrosetti, A., Rabinowitz, P.H.: Dual variational methods in critical point theory and applications. J. Funct. Anal. 14, 349-381 (1973)

3. Ambrosetti, A., Coti Zelati, V.: Periodic Solutions of Singular Lagrangian Systems. Birkhäuser, Basel (1993)

4. Ambrosetti, A., Badiale, M.: Homoclinics: Poincaré-Melnikov type results via a variational approach. Ann. Inst. H. Poincaré Anal. Non Linéaire 15, 233-252 (1998)

5. Ciesielski, J., Janczewska, J., Waterstraat, N.: On the existence of homoclinic type solutions of inhomogenous Lagrangian systems. Differ. Integral Equ. 30(3/4), 259-272 (2017)

6. Coti Zelati, V., Ekeland, I., Séré, E.: A variational approach to homoclinic orbits in Hamiltonian systems. Math. Ann. 288, 133-160 (1990)

7. Coti Zelati, V., Rabinowitz, P.H.: Homoclinic orbits for second order Hamiltonian systems possessing superquadratic potentials. J. Am. Math. Soc. 4, 693-727 (1992) 
8. Ekeland, I.: Convexity Methods in Hamiltonian Mechanics. Springer, Berlin (1990)

9. Hofer, H., Zehnder, E.: Hamiltonian Dynamics and Symplectic Invariants. Birkhäuser, Basel (1994)

10. Izydorek, M., Janczewska, J.: Homoclinic solutions for a class of the second order Hamiltonian systems. J. Differ. Equ. 219, 375-389 (2005)

11. Janczewska, J.: Almost homoclinic solutions for the second order Hamiltonian systems. Topol. Methods Nonlinear Anal. 32, 131-137 (2008)

12. Janczewska, J.: An approximative scheme of finding almost homoclinic solutions for a class of Newtonian systems. Topol. Methods Nonlinear Anal. 33, 169-177 (2009)

13. Janczewska, J.: Almost homoclinics for nonautonomous second order Hamiltonian systems by a variational approach. Bull. Belg. Math. Soc. Simon Stevin 17, 1-9 (2010)

14. Janczewska, J.: Two almost homoclinic solutions for second-order perturbed Hamiltonian systems. Commun. Contemp. Math. 14, 1250025 (2012). (9 pages)

15. Krawczyk, R.: A Note on an Approximative Scheme of Finding Almost Homoclinic Solutions for Newtonian Systems. Calculus of Variations and PDEs, pp. 107-113. Banach Center Publ., 101, Polish Acad. Sci. Inst. Math., Warsaw (2014)

16. Mawhin, J., Willem, M.: Critical Point Theory and Hamiltonian Systems. Applied Mathematical Sciences, vol. 74. Springer, Berlin (1989)

17. Montecchiari, P., Nolasco, M.: Multibump solutions for perturbations of periodic second order Hamiltonian systems. Nonlinear Anal. 27, 1355-1372 (1996)

18. Rabinowitz, P.H.: Homoclinic orbits for a class of Hamiltonian systems. Proc. R. Soc. Edinb. Sect. A 114(1-2), 33-38 (1990)

19. Rabinowitz, P.H.: Critical point theory and applications to differential equations: a survey. In: Matzeu, M., Vignoli, A. (eds.) Topological Nonlinear Analysis: Degree, Singularity, and Variations, pp. 464-513. Birkäser, Basel (1995)

20. Rabinowitz, P.H.: Variational methods for Hamiltonian systems. In: Hasselblatt, B., Katok, A. (eds.) Handbook of Dynamical Systems, vol. 1A, pp. 1091-1127. Elsevier, New York (2002)

21. Serra, E., Tarallo, M., Terracini, S.: On the existence of homoclinic motions for almost periodic second order systems. Ann. Inst. H. Poincaré Anal. Non Linéaire 13, 783-812 (1996)

Publisher's Note Springer Nature remains neutral with regard to jurisdictional claims in published maps and institutional affiliations. 